\title{
Acoustic Characterization and Modeling of the Thickness of a Submerged Tube by ANFIS and the Artificial Neural Network
}

\author{
Youssef Nahraoui \\ LMTI, Faculté des \\ sciences,Université Ibn Zohr, \\ Agadir, Maroc
}

\author{
El Houcein Aassif \\ LMTI, Faculté des \\ sciences,Université Ibn Zohr, \\ Agadir, Maroc
}

\author{
Gerard Maze \\ LOMC, Laboratoire des Ondes \\ et Milieux Complexes FRE \\ CNRS 3201, Université du \\ Havre, \\ Le Havre, France
}

\begin{abstract}
Several theoretical and experimental studies have shown that the characterization of a target (tube,...) can be made from the cut-off frequencies of the anti-symmetric circumferential waves $A_{1}$ propagating around the tube of various radius ratio $\mathrm{b} / \mathrm{a}$ ( $\mathrm{a}$ : outer radius and $\mathrm{b}$ : inner radius). This work investigates the abilities of Adaptive Neuro-fuzzy Inference System ANFIS and Artificial Neural Networks ANN to predict the thickness of a tube immersed in water for various cut-frequency of anti-symmetric circumferential wave $\mathrm{A}_{1}$. The useful data determinated from calculated trajectories of natural modes of resonances, were used to develop and to test the performances of these models. The ANN model was trained using Levenberg-Marquardt (LM) algorithm, and the ANFIS model was trained using hybrid algorithm learning that is a combination of Last Square Estimate and the gradient descent back-propagation algorithm. Several configurations are evaluated during the development of these networks. The Mean Absolute Error (MAE), Mean Relative Error (MRE), Standard Error (SE), Root Mans Square Error (RMSE) and Correlation Coefficient (R) were the statistical performance indices that were used to evaluate the accuracy of the various models. Based on the comparison between ANN and ANFIS, it was found, that the ANFIS model can be applied successfully in the in the modeling of the thickness of a Submerged Tube.
\end{abstract}

\section{Keywords}

Fuzzy logic; ANFIS; Artificial Neural Network; Acoustic response; Submerged elastic shell; Scattering waves; Circumferential waves.

\section{INTRODUCTION}

In this paper an adaptive Neuro-fuzzy Inference System ANFIS and ANN are applied to predict thickness of immersed cylindrical shell of various radius ratio b/a. Several studies have shown that the acoustic resonances of a cylindrical shell are related to its physical and geometrical properties. Conversely, from the resonances of circumferential waves we can characterize a material constituting a cylindrical shell whose geometry is known [1-6]. If the cylindrical shell is excited by a plane acoustic wave perpendicularly to its axis, the circumferential waves are generated in the shell and in the water / shell interface, these circumferential waves form standing waves on the circumference of the tube constituting resonances. The resonances of the anti-symmetric circumferential waves $\left(\mathrm{A}_{\mathrm{i}}, \mathrm{i}=0,1,2, \ldots\right.$ : index of the mode $)$ are observed on the spectrum of the acoustic pressure backscattered by the cylindrical shell [7]. Several theoretical and experimental studies show that this resonance frequencies of the circumferential waves $A_{1}$ essentially depend on the radius ratio b/a. Using the proper modes theory, we can determine the thickness of immersed cylindrical shell as a function of the cut-off frequencies of the anti-symmetric circumferential waves $A_{1}$ For a cooper cylindrical shell. The values of thickness of immersed cylindrical shell obtained from the computed values using the proper modes are used as the training data set of the ANFIS and ANN models. These models are able to predict the thickness of immersed cylindrical shell for various cut-off frequencies of the antisymmetric circumferential waves $A_{1}$ for Cooper cylindrical shell. The cut-off frequencies used, in this paper, are between 16.62 and 991.91 . The thickness values determined using the ANFIS and ANN models are compared with those determined from theoretical method to validate the robustness of the models proposed.

\section{CALCULATION OF THE FUNCTION FORM OF A CYLINDRICAL SHELL}

The diffusion of a plane wave by a cylindrical shell of radius ratio b/a filled air is studied through the solution of the wave equation and associated boundary conditions. Fig. 1 shows the fluid (1) inside the shell has a density of $\rho 1$ and propagation velocity $C_{1}$. In general, the outer fluid (2) will be different and is described by the parameters $\rho$ and $\mathrm{C}$, see table 1 .

The axis of the cylindrical shell is taken to be the z-axis of the cylindrical coordinate system $(r, \theta, z)$. Let a plane wave incident on an infinite cylindrical shell with air-filled cavity (fluid 2), be submerged in water (fluid 1), see figure 1.

The backscattered complex pressure $\mathrm{P}_{\mathrm{diff}}$ by a cylindrical shell in a faraway field is the summation of the incident wave, the reflective wave(1), surface waves tell shell waves (2) (whispering Gallery, Rayleigh, ...) and Scholte waves (A) (3) connected to the geometry of the object (fig. 2). The waves (2) and (3) are the circumferential waves: Scholte wave (A) and shell waves $\left(S_{0}, A_{1}, S_{1}, S_{2}, A_{2} \ldots\right)$. 


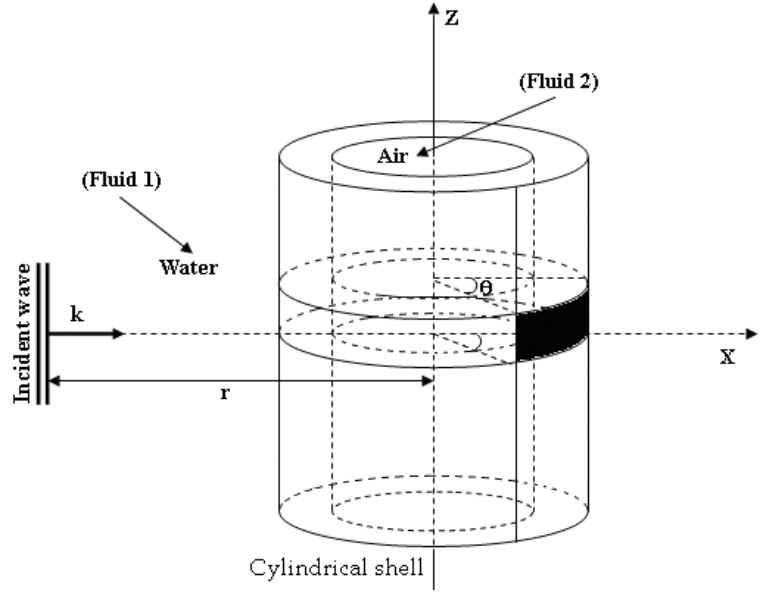

Fig 1: Geometry used for formulating the sound backscattering from a cylindrical shell

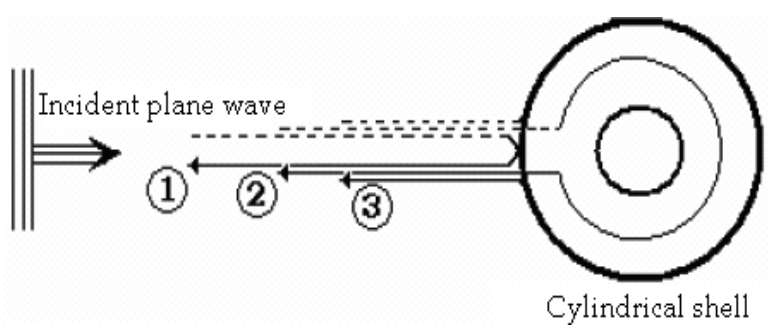

Fig 2: Mechanisms of the formation of echoes showing the specular reflection (1) and shell waves (2) and Scholte wave (A) (3).

The module of the backscattered complex pressure in a faraway field is called form function. This function is obtained by the relation [7-9]:

$$
\left|P_{\text {diff }}(\omega)\right|=\frac{2}{\sqrt{\pi k r}}\left|\sum_{n=0}^{N_{\max }} \varepsilon_{n}(-1)^{n} \frac{D_{n}^{(1)}(\omega)}{D_{n}(\omega)}\right|
$$

Where $\omega=2 \pi f$ is the angular frequency, $\mathrm{k}$ the wave number with respect to the wave velocity in the external fluid, $\varepsilon n$ is the Neumann factor $\left(\varepsilon_{\mathrm{n}}=1\right.$, if $\mathrm{n}=0 ; \varepsilon_{\mathrm{n}}=2$, if $\left.\mathrm{n}>0\right)$, $\mathrm{k}=\omega / \mathrm{c}$ is the incident wave number and $\mathrm{c}$ is the phase velocity in water, $D_{n}^{1}(\omega)$ and $D_{n}(\omega)$ are determinants computed from the boundary conditions of the problem (continuity of stress and displacement at both interfaces).

The physical parameters used in the calculation of the backscattered complex pressure are illustrated in table I.

The figure 3 shows the module of the backscattered complex pressure in function of the reduced frequency $\mathrm{x}$ (without unit) given by:

$$
x=\frac{\omega a}{c}=\frac{2 \pi}{c\left(1-\frac{b}{a}\right)} f d
$$

where $d=a-b$ is the thickness of a cylindrical shell and $f$ is the frequency of resonance of a wave in $\mathrm{Hz}$.

\begin{tabular}{|c|c|c|c|}
\hline & $\begin{array}{c}\text { Density } \\
\rho(k g / m 3)\end{array}$ & $\begin{array}{c}\text { Longitud } \\
\text { inal } \\
\text { Velocity } \\
c_{\mathbf{L}_{\mathbf{L}}(\mathrm{m} / \mathrm{s})} \\
\end{array}$ & $\begin{array}{c}\text { Transver } \\
\text { se } \\
\text { Velocity } \\
c_{T}(\mathrm{~m} / \mathbf{s}) \\
\end{array}$ \\
\hline Cooper & 8930 & 4760 & 2325 \\
\hline Water & 1000 & 1470 & $\begin{array}{r}-------- \\
---\end{array}$ \\
\hline Air & 1.29 & 334 & $\begin{array}{c}------------ \\
---- \\
\end{array}$ \\
\hline
\end{tabular}

Table 1: Physical parameters

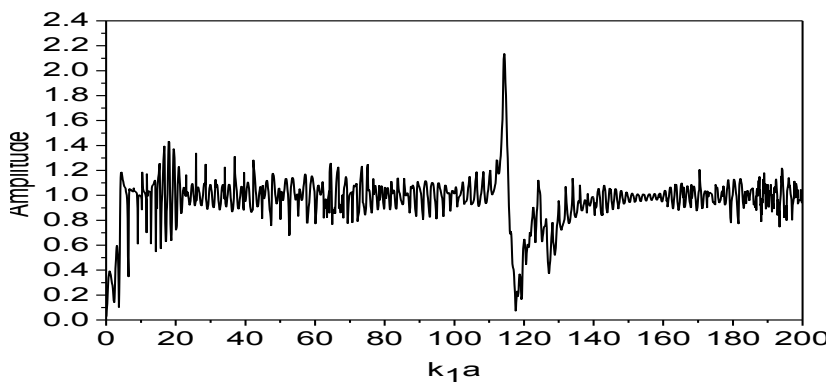

Fig 3: Spectre de résonance d'un tube en cuivre de rapport de rayons $b / a=0.92$

\section{DETERMINATION OF THICKNESS OF IMMERSED TUBE USING THE PROPER MODES THEORY}

According to the similarity between the circumferential waves in the case of a thin elastic tube and the Lamb waves in the case of a plaque of the same thickness, the classical relations of the Lamb waves remains valid to calculate the values of the Tickness of a Submerged Tube as a function of cut-off frequency of circumferential waves in the case of a tube which are obtained by the formula (3) [2-5, 10-13]:

For A1 mode:

$$
\left(\frac{a-b}{a}\right)=\frac{\pi \cdot c_{T}}{c \cdot(x)_{c}^{A 1}} .
$$

Where $d=a-b$ is the thickness of a cylindrical shell $(a=1 \mathrm{~m}$ : outer radius (in our case) and $\mathrm{b}$ : inner radius) and ( $\mathrm{x}) \mathrm{c}$ is the cut-off frequencies of circumferential waves $A_{1}$ without unity.

\section{MATERIALS AND METHOD}

\subsection{Architecture of ANN Model}

The networks used in the present study were feedforward and possessed two or three layers of processing units with continuous-valued outputs. Fig. 4 shows a typical neural network consisted of input, sum function, sigmoid activation function and output. This type of neural network follows an advanced learning algorithm called Levenberg-Marquardt back-propagation that requires both input and output data, is used. The input values to a neuron are obtained by multiplying the output of the connected neuron by the synaptic strength of the connection between them. The weighted sums of the input components $\mathrm{x}_{\mathrm{i}}$ are calculated by using the following equation $[14,15]$ :

$$
\text { (y) }{ }_{j}=f\left(\sum_{i=1}^{l} w_{i j} x_{i}+b\right)
$$


where $(y)_{j}$ is the output from neuron, $\mathrm{w}_{\mathrm{ij}}$ is connection's weight, $b$ is bias weight, $n$ is the number of neurons or processing elements (PE) in each layer and $f$ is the activation function. In this paper, the log-sigmoid used as activation function as follows $[14,15]$ :

$$
O_{j}=f\left((y)_{j}\right)=\frac{1}{1+\exp \left(-(y)_{j}\right)}
$$

After processing all of the layers, the activated result of the output layer $\mathrm{O}_{\mathrm{j}}$, compared with the desired output $\mathrm{y}_{\mathrm{t}}$, and the resulted error will be propagated backward the network's weight that are adjusted during the learning process to minimize the error, defined as the difference between the calculated output and the desired output. This process is usually performed by a method known as error backpropagation (BP) method. In this work, Levenberg-Marquardt back-propagation (LMBP) algorithm is used as training algorithm instead of commonly used standard BP method for its robustness in computing process [16].

\subsection{ANN model and training phase}

Neural network focuses on supervised learning algorithms. These learning algorithms are characterized by the usage of a given output that is compared to the predicted output and by the adaptation of all parameters according to this comparison. The parameters of a neural network are its weights. All weights are usually initialized with random values drawn from a standard normal distribution;

Fig. 4 shows a three-layer neural network. All the units are formed into multiple layers, i.e., an input layer, a hidden layer, and an output layer. The square error of the $\mathrm{p}_{\mathrm{th}}$ training pattern Ep is defined as:

$$
E_{P}=\frac{1}{2} \sum_{k=1}^{m}\left(T_{p k}-O_{p k}\right)^{2}
$$

where $T_{p k}$ is target output to the kth output unit for $\mathrm{p}_{\mathrm{th}}$ training pattern, $\mathrm{O}_{\mathrm{pk}}$ is the output signal to the kth output unit for $\mathrm{p}_{\mathrm{th}}$ training pattern, and $\mathrm{m}$ is the number of output units. In the training process, $\mathrm{w}_{\mathrm{ij}}$ and $\mathrm{b}$ are modified repeatedly based on gradient descent method to minimize the above error. Through such an iterative process, the network attains the ability to promptly output the similar signal to the teacher's one. This training algorithm is called back propagation neural network.

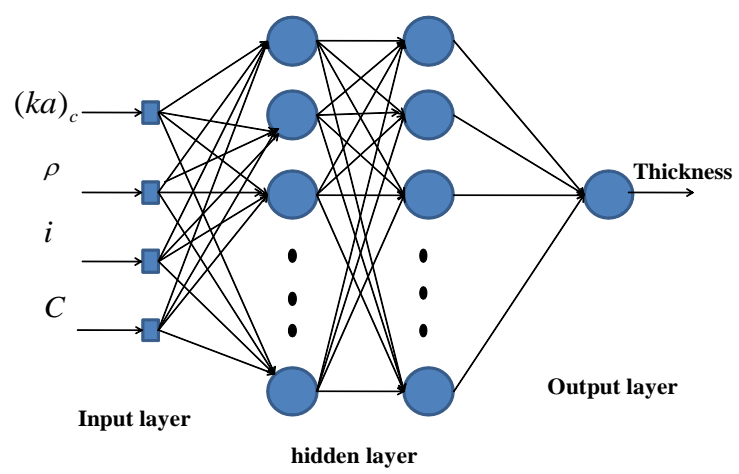

Fig 4: PML to four entries $\rho$ :material density, $b / a$ : radius ratio, $i$ : index of antisymmetric waves $A_{i}, C$ :velocities of propagation in the material $\left(C_{T}\right.$ : velocity of shear wave; or $C_{L}$ : velocity of longitudinal wave).

\section{FUZZY INFERENCE SYSTEM}

Fuzzy inference systems (FIS) is one of the most famous applications of fuzzy logic and the theory of fuzzy sets, proposed by L. Zadeh in 1965 [38]. The success of FIS is their following two main features. On one hand, they are able to manipulate language concepts. On the other, they are universal approximators able to reproduce nonlinear transformations between inputs and outputs. The fuzzy models are means of capturing human's expert knowledge about the process, in terms of fuzzy (if-then) rules. It can model the qualitative aspects of human knowledge and reasoning processes without employing precise quantitative analyses.

The fuzzy inference system is composed of four functional elements, as follows [17-19]:

1. A knowledge base that contains definitions of fuzzy sets and fuzzy operators;

2. An inference mechanism that performs all calculations;

3. A fuzzification inference, which transforms the crisp inputs into degree of match with linguistic values.

4. A defuzzification inference, which transforms the fuzzy results of the inference into a crisp output.

Several types of FIS have been proposed in the literature [20], which, vary due to differences between the specification of the consequent part and the defuzzification schemes. This paper incorporates one of these types called Takagi-Sugeno FIS [21], to propose a systematic scheme for the development of fuzzy rules using the input/output data sets.

A typical fuzzy rule in a Sugeno fuzzy model has the format:

If $x$ is $A$ and $y$ is $B$ then $z=f(x, y)$

where A and B are fuzzy sets in the antecedent; $\mathrm{z}=\mathrm{f}(\mathrm{x}, \mathrm{y})$ is a crisp function in the consequent. When $\mathrm{f}(\mathrm{x}, \mathrm{y})$ is a first order polynomial, we have the first-order sugeno fuzzy model. When $\mathrm{f}$ is a constant, we have the zero-order Sugeno fuzzy model. Consider first-order Sugeno fuzzy inference systems which contain two rules:

Rule 1: if $\mathrm{x}$ is $A_{1}$ and $y$ is $B_{1}$ then $f_{1}=p_{1} x+q_{1} y+r_{1}$.

Rule 2: if $\mathrm{x}$ is $A_{2}$ and $\mathrm{y}$ is $B_{2}$ then $f_{2}=p_{2} x+q_{2} y+r_{2}$.

Weighted averages are used in order to avoid complexity in deffuzification processes. Fig.5 illustrates graphically the fuzzy reasoning mechanism to derive an output $f$ from a given input vector $(\mathrm{x}, \mathrm{y})$. The firing strengths $\omega_{1}$ and $\omega_{2}$ are usually obtained as the product of the membership grades in the premise part, and the output $f$ is the weighted average of each rule's output. To facility the learning of the sugeno fuzzy model, into the framework of adaptative networks we can compute gradient vectors systemically. The resultant network architecture is called Adaptative Neuro Fuzzy Inference system (ANFIS).

\section{ADAPTIVE NEURO-FUZZY INFERENCE SYSTEM ARCHITECTURE}

The Adaptive Network-based Fuzzy Inference System (ANFIS) has been widely used in modelling, it is developed by Jang in 1993 [17]. Given an input/output data set, the ANFIS can construct a Fuzzy Inference System whose 
membership function parameters were adjusted using a hybrid algorithm learning that is a combination of Last Square Estimate and the gradient descent back-propagation algorithm or other similar optimisation technique. This allows Fuzzy system to learn from the data they are modelled.

For simplicity, we assume the FIS with two input, $\mathrm{x}$ and $\mathrm{y}$ with one response $f$. From the first-order Sugeno fuzzy model, a typical rule set with two fuzzy if-then rules can expressed as below. The architecture is shown in Fig.6. Generaly, an ANFIS model consistes of five layers.

Layer 1: Input variables are fuzzificated and the membership grade of a linguistic label. For instance, the node function of the ith node may be generalized bell membership function:

$$
\mu_{A_{i}}=\frac{1}{1+\left[\frac{x-c_{i}}{a_{i}}\right]^{b_{i}}}
$$

where $\mathrm{x}$ is the input to node $\mathrm{i} ; A_{i}$ is the linguistic label (small, large, etc.) associated with this node; and $\left\{a_{i}, b_{i}, c_{i}\right\}$ is the parameter set that changes the shapes of the membership function. Parameters in this layer are referred to as the premise parameters.

Layer 2: The function is T-norm operator that performs the firing strength of the rule, e.g., fuzzy conjective AND and OR. The simplest implementation just calculates the product of all incoming signals.

$$
\omega_{i}=\mu A_{i}(x) \mu B_{i}(\mathrm{y}), \mathrm{i}=1,2
$$

Layer 3: Every node in this layer is fixed and determines a normalized firing strength. It calculates the ratio of the ratio of the $j_{\text {th }}$ rule's firing strength to the sum of all rules firing strength.

$$
\varpi_{i}=\frac{\omega_{i}}{\omega_{1}+\omega_{2}}, \quad \mathrm{i}=1,2
$$

Layer 4: The nodes in this layer are adaptive are connected with the input nodes and the preceding node of layer 3 . The result is the weighted output of the rule $j$.

$$
\varpi_{i} f_{i}=\omega_{i}\left(p_{i} x+q_{i} y+r_{i}\right)
$$

where $\omega_{i}$ is the output of layer 3 and $\left\{p_{i}, q_{i}, r_{i}\right\}$ is the parameter set. Parameters in this layer are referred to as the consequent parameters.

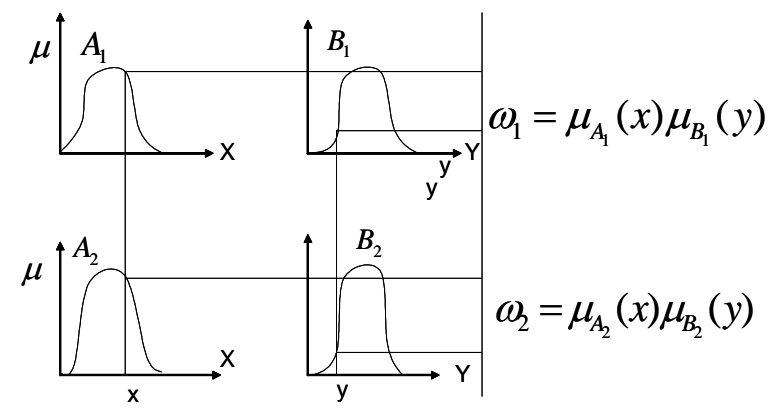

Fig 5: First-order Sugeno fuzzy model
Layer 5: This layer consists of one single node which computes the overall output as the summation of all incoming signals.

$$
\text { Overall Output } \sum_{i} \varpi_{i} f_{i}=\frac{\sum_{i} \omega_{i} f_{i}}{\sum_{i} \omega_{i}}
$$

The constructed adaptive network in figure 6 is functionally equivalent to a fuzzy inference system in figure 4 .

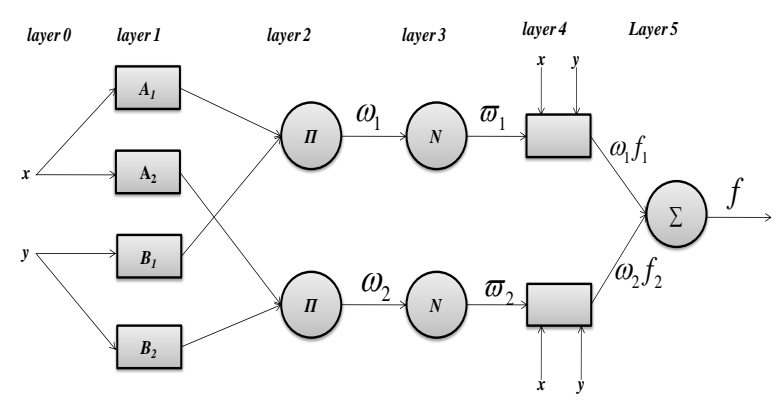

Fig 6: ANFIS architecture

\section{COLLECTION OF DATA}

As stated earlier, the objective of this work was to evaluate of ANN and ANFIS for prediction of the thickness of a tube immersed in water. To accomplish this objective, a data base is collected to involve and test the performance of these models starting from the results determined from the values calculated using the eigenmode theory of resonances of the antisymetric circumferential wave $A_{1}$. The density of material, the cut-frequency of anti-symmetric circumferential wave $A_{1}$, and longitudinal and transverse velocities, of the material constituting the cylindrical shell, are retained like relevant entries of the model because these parameters characterize the cylindrical shell and the types of circumferential waves propagating around this one. The thickness of a cooper cylindrical shell constitutes the output of fuzzy system and neural network.

\subsection{Training of the ANN model}

In this work, the perceptron multilayers (PML) is used as a model of network architecture to predict the thickness of a tube immersed in water.

Before any training, it's it is essential to normalize all the variables of entries to remain in the linear part of the sigmoidal function, the input and output data are normalized between 0.1 and 0.9 using the following equation [22]:

$$
y=0.1+0.8 \times\left(\frac{x-x_{\min }}{x_{\max }-x_{\min }}\right)
$$

Where $\mathrm{x}_{\max }$ and $\mathrm{x}_{\min }$ are maximum and minimum values of a particular parameter in the entire data set, respectively.

They are the weights of these connections that control the operation of the network [23].

In this work, the number of neurons in hidden layers varies from 1 to 8 ; the architecture of this network is represented in Fig. 8. The optimal configuration is obtained by the minimization of the difference between the desired and the predicted outputs. 
The collected data are divided into two sets. The training and the validation sets are made up, respectively, of $2 / 3$ and $1 / 3$ of the collected data base [24]. These proportions of the training and validation bases are selected randomly from the data base. The Levenberg-Marquated back-propagation algorithm using the supervised training technique is used to involve the hidden layers of the artificial neural network model [25], [26].The sigmoid tangent function is regarded as transfer function of the neurons. It undergoes sharp variations when the parameter values are high for the hidden neurons entries. Not to leave the linear part of the sigmoid, the synaptic weights are initialized randomly.

\subsection{Training of the ANFIS model}

ANFIS networks method requires for its training a dataset of the thickness of a cylindrical shell calculated by the analytical method or obtained by experiments. This dataset is divided into two sets. The first $2 / 3$ training data set was used for training the ANFIS while the remaining $1 / 3$ checking data set were used for validating the identified model, the number of membership function is fixed to $7 \mathrm{MF}$, so the rule number is 7. The ANFIS used here contains a total number of parameters: 35, of which number of linear parameters: 14 and number of nonlinear parameters: 21. The desired and predicted values for both training data and checking data are essentially the same in fig 11 and 12 . The architecture of this network is represented in Fig. 7

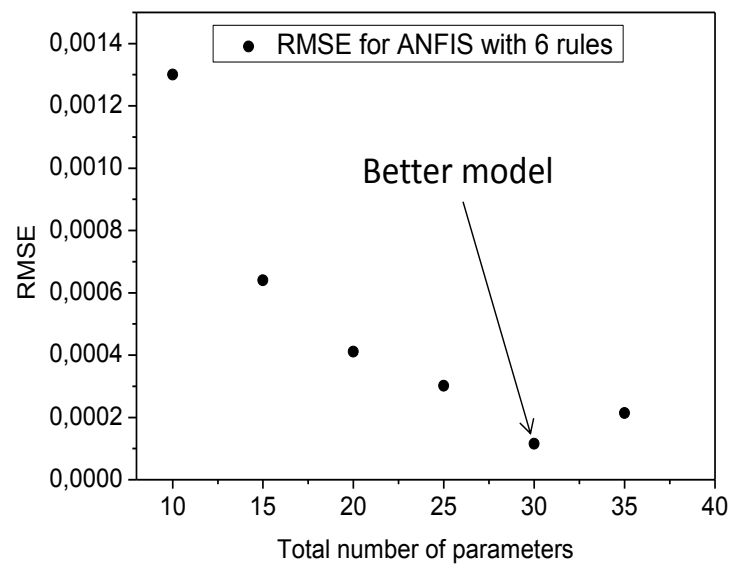

Fig 7: Errors for the prediction of the Tickness of a Submerged Tube by ANFIS with different ANFIS configuration

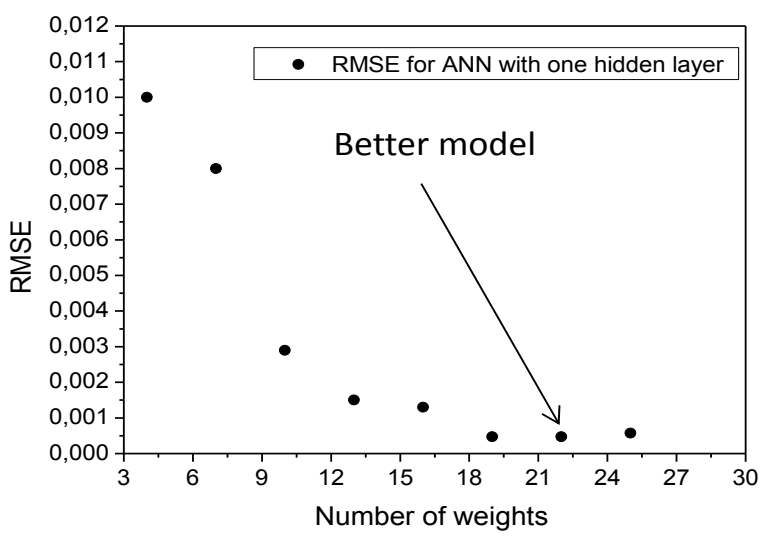

Fig 8: Errors for the prediction of the Tickness of a Submerged Tube by ANN with different ANN configuration

\section{RESULTS AND DISCUSSION}

The performance of ANFIS and ANN models for a set of data in both training and testing phases were evaluated according to statistical criteria such as the correlation coefficient $\mathrm{R}$, MAE, MRE, SE, and the root error root mean square (RMSE). The selection of different models is performed based comparison of different error MAE, MRE and SE between the values of thickness predicted by ANFIS and ANN and those desired. The correlation coefficient $\mathrm{R}$ and $\mathrm{R}^{2}$ of the linear regression of the determination are used as measures of the performance of the model between the predicted values and the desired values. The various measures of error and the correlation coefficient are given by the following relationships:

$$
\begin{gathered}
M A E=\frac{1}{n} \sum_{i=1}^{n}\left|D_{i}-P_{i}\right| \\
M R E=\frac{1}{n} \sum_{i=1}^{n} \frac{\left|D_{i}-P_{i}\right|}{D_{i}} \\
R=1-\frac{\sum_{i=1}^{n}\left(D_{i}-P_{i}\right)^{2}}{\sum_{i=1}^{n}\left(D_{i}-P_{m}\right)^{2}} \\
S E=\frac{\sqrt{\sum_{i=1}^{n}\left(D_{i}-P_{i}\right)^{2}}}{n-1}
\end{gathered}
$$

where $\mathrm{n}$ is the number of data, $\mathrm{P}_{\mathrm{i}}$ and $\mathrm{D}_{\mathrm{i}}$ is the predicted and desired of the thickness of immersed tube respectively and $P_{m}$ is the mean of predicted values.

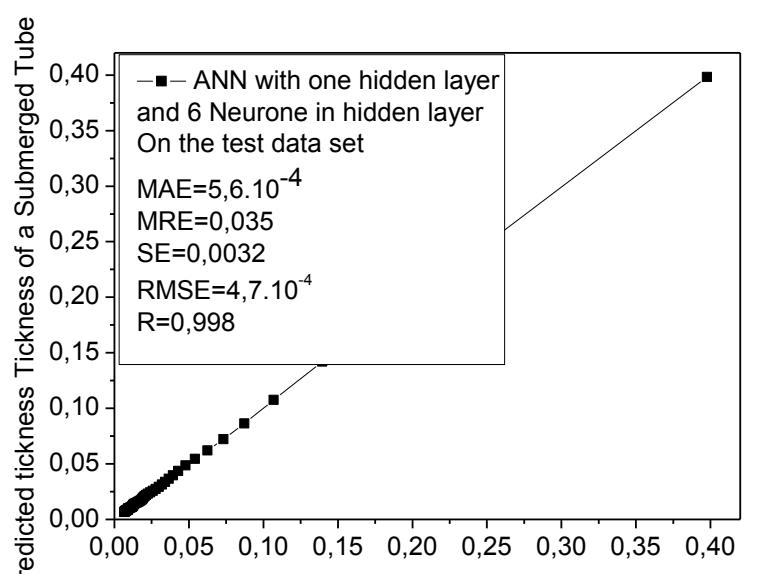

Fig 9: correlation between the thickness of immersed tube calculated by the theoretical method and that predicted by ANN 


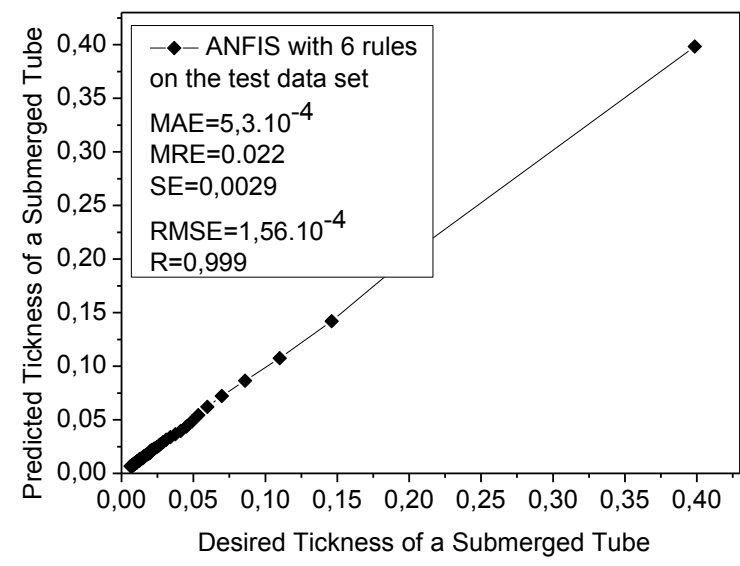

Fig 10: correlation between the thickness of immersed tube calculated by the theoretical method and that predicted by ANFIS

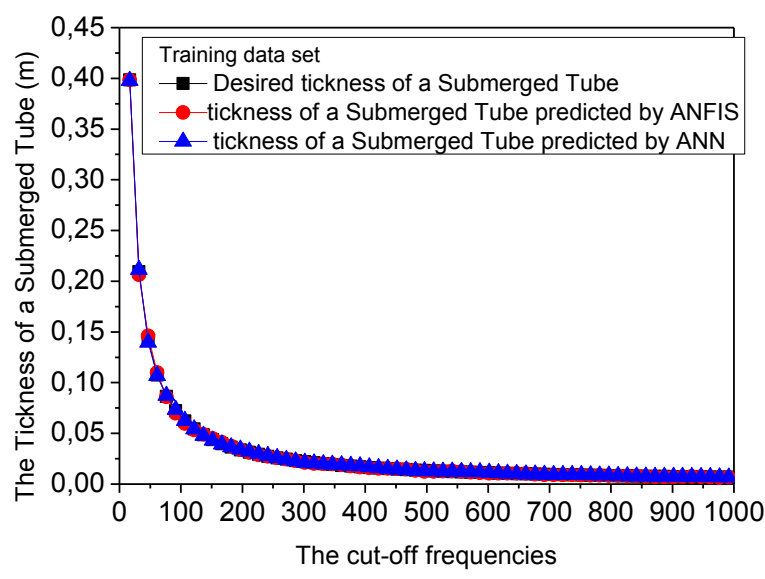

Fig 11: training dataset (wave $A_{1}$ ) represents the evolution of the thickness of immersed tube as a function of frequency calculated by the theoretical method and that predicted by ANFIS and ANN

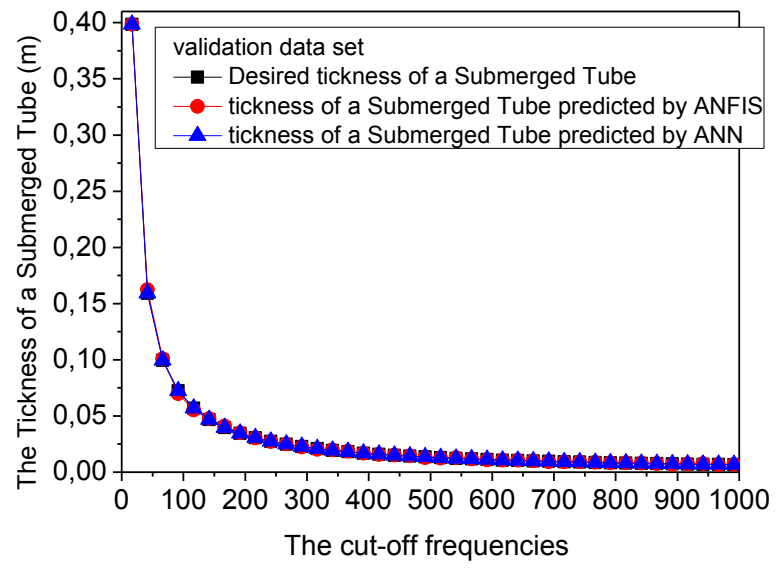

Fig 12: validation dataset (wave $A_{1}$ ) represents the evolution of thickness of immersed tube as a function of frequency calculated by the theoretical method and that predicted by ANFIS and ANN

The correlation coefficient is a statistical criterion commonly used and which provides information about the strength of the linear relationship between the observed and the calculated values. The performance of ANFIS and ANN models of the training and test data are represented in Fig. 9 and 10.

The analysis is repeated several times. Indeed, the error values are measured for each ANFIS and ANN architecture based on the number of rules and the type of the membership function used and that of neural network is based on the number of neurons in the hidden layers.

\section{CONCLUSION}

The potential of ANFIS and ANN modeling methods for estimation of the thickness of a tube immersed in water for various cut-frequency of ant-symmetric circumferential wave $\mathrm{A}_{1}$ with the minimum of input data was explored in this paper. This work show that modelling of the thickness of a tube immersed is best estimation through of ANFIS approaches. The optimal trained model taking into account some characteristics of the tube can be used as an alternative way in the modelling behaviour system. In this article, these models are used like new nondestructive measurement technique to determine thickness of a tube immersed in water from cutfrequencies of the acoustic circumferential waves propagating around the tube. The use of adaptive Neuro-fuzzy Inference System ANFIS does not present any approximation as in the case of the natural modes method which assimilates the tubes to the plates with the same thickness and that is not sullied with errors as in the case of the time-frequency representations of Wigner-Ville that determines the Thickness of a Submerged manually starting from the time-frequency image. These models can be used also to predict the evolution of the group and phase velocities according to the frequency. It also can constitute a help for the estimate of various parameters of a tube starting from the characteristics of which it is disposed

\section{REFERENCES}

[1] Y. Nahraoui, E. Assif, G. Maze, R. Latif, “Modelling the Cut-off Frequency of Acoustic Signal with an Adaptative Neuro-Fuzzy Inference System (ANFIS)', IJACSA. Vol.4, No. 6, pp. 23-33, (2013)

[2] R. Latif, E. Aassif, M. Laaboubi, G. Maze, "Détermination de l'épaisseur d'un tube élastique à partir de l'analyse temps-fréquence de Wigner-Ville", Acta Acustica united with Acustica, Vol. 95, pp. 253-257, (2009)

[3] M. Talmant, J. L. Izbicki, G. Maze, G. Quentin J. Ripoche. "External wave resonance on thin cylindrical shells". J. Acoustique, 4, pp. 509-523(1991).

[4] L. Haumesser, D. Décultot, F. Léon, and G. Maze, "Experimental identification of finite cylindrical shell vibration modes", Journal of the Acoustical Society of America, Vol. 111, 5, pp. 2034-2039, (2002)

[5] J. D. N. Cheeke, X. Li, and Z. Wang, "Observation of flexural Lamb waves (A0 mode) on water-filled cylindrical shells", Journal of the Acoustical Society of America, Vol. 104, 6, pp. 3678-3680, (1998).

[6] P. L. Marston and N. H. Sun, "Backscattering near the coincidence frequency of a thin cylindrical shell: Surface wave properties from elastic theory and an approximate ray synthesis," J. Acoust. Soc. Amer., vol. 97, pp. 777 783, 1995.

[7] G. Maze, "Acoustic scattering from submerged cylinders. MIIR Im/Re: Experimental and theoretical 
study," J. Acoust. Soc. Amer., vol. 89, pp. 25592566,1991

[8] R. Latif, E. Aassif, G. Maze, A. Moudden, B. Faiz, "Determination of the group and phase velocities from time-frequency representation of Wigner-Ville", Journal of Non Destructive Testing \& Evaluation International, Vol.32, 7, pp. 415-422, (1999)

[9] G. Maze, J. Ripoche, A. Derem, J. L. Rousselot, "Diffusion d'une onde ultrasonore par des tubes remplis d'air immergés dans l'eau", Acustica, vol. 55, pp. 69-85, (1984).

[10] R. LATIF, E. AASSIF, G. MAZE, D. DECULTOT, A. MOUDDEN, B. FAIZ, "Analysis of the circumferential acoustic waves backscattered by a tube using the timefrequency representation of Wigner-Ville", Journal of Measurement Science and Technology, Vol. 11, 1, pp. 83-88, (2000).

[11] R. LATIF, E. AASSIF, A. MOUDDEN, B. FAIZ, "Caractérisation ultrasonore d'un matériau élastique à partir de l'analyse de l'image temps-fréquence de Wigner-Ville ", Acta Acustica united with Acustica, Vol. 89, pp. 253-257, (2003).

[12] Younho Cho, "Estimation of ultrasonic guided wave mode conversion in a plate with thickness variation", Ultrasonics, Ferroelectrics and Frequency Control, IEEE Transactions, Vol. 47, 3, pp. 591-603, (2000).

[13] Atkinson D., Hayward G., "Embedded acoustic fibre wave guides for Lamb wave condition monitoring", Ultrasonics Symposium, IEEE, Vol. 1, pp. 699-702, (1999).

[14] Sarıdemir M. Predicting the compressive strength of mortars containing metakaolin by artificial neural networks and fuzzy logic. Adv Eng Soft 2009;40(9):920 7.

[15] Fausett LV. Fundamentals of neural networks: architectures, algorithms, and applications. Prentice Hall; 1994.
[16] Suratgar AA, Tavakoli MB, Hoseinabadi A. Modified Levenberg-Marquardt method for neural networks training. World Acad Sci Eng Technol 2005;6:46-8.

[17] Jang J-SR. ANFIS: Adaptive-network-based fuzzy inference systems. IEEE Trans Syst Man Cybern 1993; 23(3):665-85.

[18] S. Horikawa, T. Furuhashi, and Y Uchikawa. IEEE Trans. Neural Networks 3:801-806, 1992.

[19] H. Ishibuchi, R. Fujioka, and H. Tanaka. IEEE Trans. Fuzzy Systems 1:85-97, 1993.

[20] Lee CC. Fuzzy logic in control system: Fuzzy logic controller-part I and part II. IEEE Trans Syst Man Cybern 1990; 20(2):404-35.

[21] Sugeno M. Industrial applications of fuzzy control. New York: Elsevier Ltd.; 1985.

[22] S. K. Singh, K. Srinivasan, and D. Chakraborty, "Acoustic characterization and prediction of surface roughness," J. Mater. Processing Technol., vol. 152, pp. 127-130, 2004.

[23] G. Dreyfus, J. M. Martinez, M. Samuelides, M. B. Gordon,F. Badran, S. Thiria, and L. H'erault, R'eseaux de Neurones Méthodologie et Applications. 2nd ed. Paris, France: Eyrolles, 2004. (in French)

[24] I. J. Leontaritis and S. A. Billings, "Model selection and validation methods for non linear systems," J. Contr., vol. 45, pp. 349-359, 1997.

[25] D. Plaut, S. Nowlan, and G. E. Hinton, "Experiments on learning by back propagation," Technical Report CMUCS-86-126, Carnegie-Mellon University, Pittsburgh, PA, 1986.

[26] D. E. Rumelhart, G. E. Hinton, and R. J. Williams, "Learning internal representations by error back propagation," in Parallel Distributed Processing: Explorations in the Microstructure of Cognition. vol. 1, Cambridge, MA: MIT Press, 1986, pp. 318- 362. 